\title{
TUR Syndrome
}

Uncommonly, after transurethral surgery the patient may become confused, uncooperative and hypotensive, and develop a brachycardia, sickness and sometimes collapse. This syndrome may be associated with hyponatraemia and hypervolaemia (and possibly hyperammonaemia) and is thought to be due to the absorption of isotonic irrigating fluid during the course of operation and in the post-operative period. The hyperammonaemia results from the metabolic breakdown of absorbed glycine and may be partly responsible for signs of encephalopathy.

The syndrome is rapidly corrected when the irrigation is discontinued, diuretics are prescribed and the sodium deficit is corrected by an infusion of $\mathrm{N}$ or $2 \mathrm{~N}$ saline (although the latter is rarely needed). 